\title{
Operational Modal Analysis on a 3D Scaled Model of a 3-Storey Aluminium Structure
}

\author{
M. Fadhil Shazmir ${ }^{1}$, N. Ayuni Safari ${ }^{2}$, M. Azhan Anuar*3, A.A.Mat Isa ${ }^{4}$, Zamri A.R ${ }^{5}$ \\ ${ }^{12345}$ Department of Mechanical Engineering, University Teknologi MARA, 40450 Selangor, Malaysia \\ *Corresponding author E-mail:azhan788@salam.uitm.edu.my
}

\begin{abstract}
Obtaining a good experimental modal data is essential in modal analysis in order to ensure accurate extraction of modal parameters. The parameters are compared with other extraction methods to ascertain its consistency and validity. This paper demonstrates the extraction of modal parameters using various identification algorithms in Operational Modal Analysis (OMA) on a 3D scaled model of a 3-storey aluminium structure. Algorithms such as Frequency Domain Decomposition (FDD), Enhanced Frequency Domain Decomposition (EFDD) and Stochastic Subspace Identification (SSI) are applied in this study to obtain modal parameters. The model test structure is fabricated of aluminium and assembled using bolts and nuts. Accelerometers were used to collect the responses and the commercial post processing software was used to obtain the modal parameters. The resulting natural frequencies and mode shapes using FDD method are then compared with other OMA parametric technique such as EFDD and SSI algorithm by comparing the natural frequencies and Modal Assurance Criterion (MAC). Comparison of these techniques will be shown to justify the validity of each technique used and hence confirming the accuracy of the measurement taken.
\end{abstract}

Keywords: Operational Modal Analysis, Frequency Domain Decomposition, Dynamic characteristics, Stochastic Subspace Identification, Enhanced Frequency Domain Decomposition, 3D Aluminium Structure

\section{Introduction}

Structural Health Monitoring (SHM) and Vibration-Based Maintenance are becoming more important due to its versatility and without distraction in the operation of the structure or machinery. It requires System Identification (SI) analysis in determining modal parameters of a structure. The first step in SHM normally start with the identification of structure natural frequency, mode shape and damping value.

The amount of research related to condition monitoring has increased significantly in the past decades since conditional assessment of a large structures become important around the world due to its rapid increase in development of construction industry. Damage in structures not only defined by the defect or flaw but also structures are not operating at it optimum operation condition. Normally damage need to be detected at early stage for condition monitoring using the available technology as it is always motivated by the life-safety and also the economy effect.

Many techniques have been developed in SHM to conditioning monitoring especially in detecting damage on different structure elements. SHM using global technique namely vibration-based damage detection, is usually used to monitor modal properties such as monitors the changes in natural frequencies, damping loss factor and mode shapes. SHM techniques and models have been established and applied in aerospace structures [1], [2] and also civil structures such as building [3]-[5], and bridges [6]-[9].

The system's performance will be affected over a period of time or immediately depends on the size of the crack, the location of the crack and also the loading conditions applied to the system. Experimentally, there are two ways to identify the dynamic characteristics of any structures namely Experimental Modal Analysis
(EMA) and Operational Modal Analysis (OMA). EMA is a classical approach that requires an input excitation and it must be measured [10]. In contrast to Operational Modal Analysis (OMA) where output only response is measured.

In OMA technique, the output signals is measured from a structure which is directly or randomly excited in an actual ambient condition from operating forces as an unmeasured input. This technique provides a much needed tool for the determination of the dynamic characteristics of large and complex structures or mechanisms especially when input forces cannot be directly controlled or measured.

This paper will discuss and analyzes dynamic characteristic of a 3D scaled building model of a 3-storey aluminium structure by obtaining acceleration data then processed through System Identification (SI) method which in this case is Operational Modal Analysis. Five (5) different algorithms in OMA both parametric and non-parametric method were used to obtain the modal parameters of the tested structure. The algorithms used were Frequency Domain Decomposition (FDD), Enhanced Frequency Domain Decomposition (EFDD) and Stochastic Subspace Identification (SSI).

\section{Operational Modal Analysis Techniques}

Different algorithms in OMA were used to identify the modal parameters and were compared to indicate their relative accuracy /validity. They are Frequency Domain Decomposition (FDD), Enhanced Frequency Domain Decomposition (EFDD) and Stochastic Subspace Identification (SSI) techniques finally were done for modal determination. 


\subsection{Frequency Domain Decomposition}

Frequency Domain Decomposition (FDD) which is also known as non-parametric method is an extension of the Basic Frequency Domain (BFD) technique [11]. In this technique, the vibrational modes can be obtained from spectral densities calculated in which case the modal parameters are estimated directly from the signal processing calculations. The modes are constructed using Singular Value Decomposition (SVD) of each Power Spectral Density (PSD) matrix generated. In Operational Modal Analysis (OMA), there are three identification algorithms method applied in this study. Frequency domain Decomposition (FDD) method was chosen as a reference to compare with other identification algorithms since FDD is well known as one of the reliable and more user friendly for operational modal analysis of structures [14].

\subsection{Enhanced Frequency Domain Decomposition}

In contrast to FDD, Enhanced Frequency Domain Decomposition (EFDD) is parametric technique. It computes the auto and crosscorrelation functions in order to extract the frequency and damping of a particular mode [11]. The PSD which is identified around a peak of resonance is converted to time domain using the Inverse Discrete Fourier Transform (IDFT). In this case, the numbers of zero crossing times are required in order to obtain the respective natural frequencies. The damping ratio is then obtained using the logarithmic decrement of the corresponding single degree of freedom (SDOF) of the normalized auto correlation function.

\subsection{Stochastic Subspace Identification}

Similar EFDD, this is another parametric method where in this technique modal parameters are estimated by utilizing a parametric model fitted to the signal processed data. The relevant modal parameters are identified based on the output-only covariancedriven and data-driven SSI [12] where correlation function is used in covariance-driven SSI method while raw time data is used in data-driven SSI method. The standard three identification classes for SSI method namely Unweighted Principle Components (UPC), Principle Components (PC) and Canonical Variate Analysis (CVA) can be employed for the modal identifications.

\section{Materials and Methods}

The Data Acquisition Systems (DAQ) used in this study is Bruel\&Kjaer PULSE ${ }^{\mathrm{TM}}$ Multi-Analyzer System and the structure is made of aluminium with dimension $(850 \mathrm{~mm} \times 250 \mathrm{~mm} \times$ $250 \mathrm{~mm}$ ). Post processing of the random acceleration signal data obtained from measurement is exported into the Ambient Response Testing and Modal Identification Software (ARTeMIS ${ }^{\mathrm{TM}}$ ) and required for Fourier transform of the correlation function in order to estimate Power Spectral Density (PSD) matrix

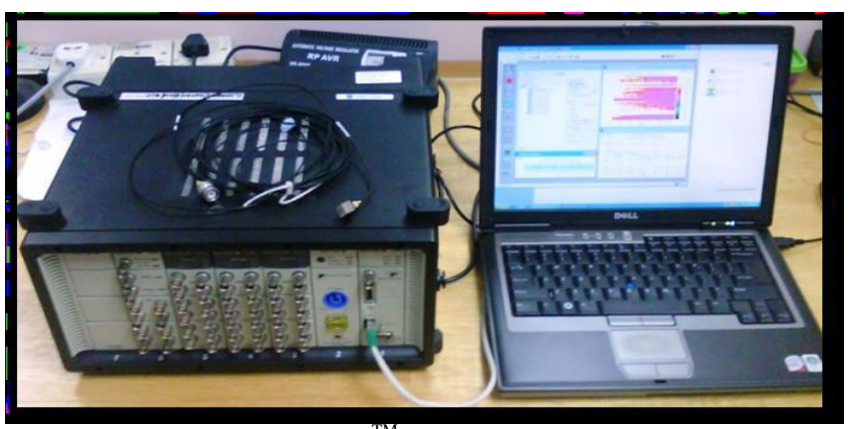

Fig. 1: The Bruel\&Kjaer PULSE ${ }^{\mathrm{TM}}$ Multi-Analyzer System connected to a computer with The Bruel\&Kjaer PULSE ${ }^{\mathrm{TM}}$ Multi-Labshop software and ARTeMIS $^{\mathrm{TM}}$ software.

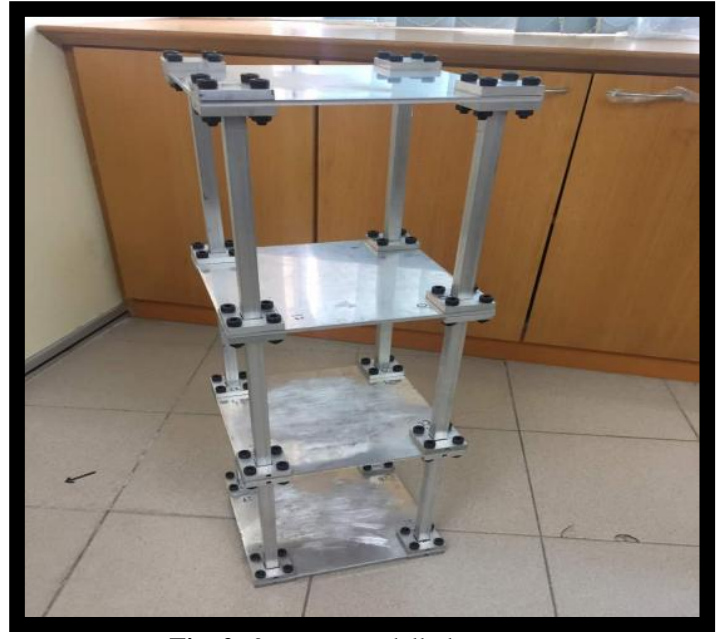

Fig. 2: 3-storey modelled structure

For this study, 14 accelerometers (4 references with 10 roving) were used with two data sets to compensate for 24 DOF's. The structure was excited by randomly and continuously tapping with relatively enough energy to excite all frequencies of interest. Preliminary testing of scrubbing the structure with metal bars introduced too much noise modes both for parametric and nonparametric techniques. Bruel\&Kjaer PULSE ${ }^{\mathrm{TM}}$ Multi-Labshop Software was used to set up the hardware, create the geometry, assign measurement points and perform the test. The raw data which consists of geometrical values and series of measurements are then directly exported to the ARTeMIS ${ }^{\mathrm{TM}}$ software for signal processing calculation and modal extraction. Frequency Domain Decomposition (FDD), Enhanced Frequency Domain Decomposition (EFDD) and Stochastic Subspace Identification (SSI) techniques are performed after all data are exported.

\section{Results and Discussion}

The first step in considering the results obtained from this study is by cross referencing the FDD modes obtained from the modeled structure with the FDD modes obtained by [13]. This comparison is made to look at the modes consistency for the similar type of structure. It is understood that the structure's natural frequencies cannot be compared as the total mass and material of both structures are different. On the other hand, the mode shapes could be compared visually in reference to each other as both of the structure are tested under the same boundary condition. This initial checking of the visual mode shapes obtained in this study will act as a validation to ensure that FDD result could be used as further reference when compared with the other parametric methods.

\subsection{FDD and EFDD Methods Peak Picking Identifica- tion}

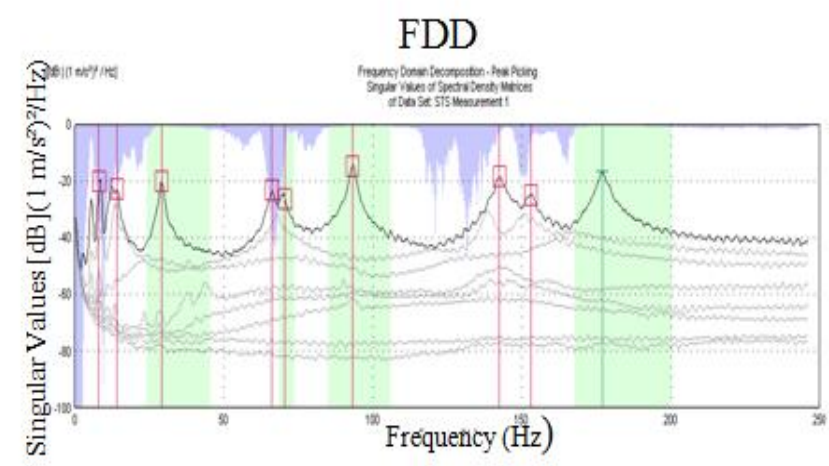

Fig. 3: FDD Singular Value Decomposition (SVD) Plot 


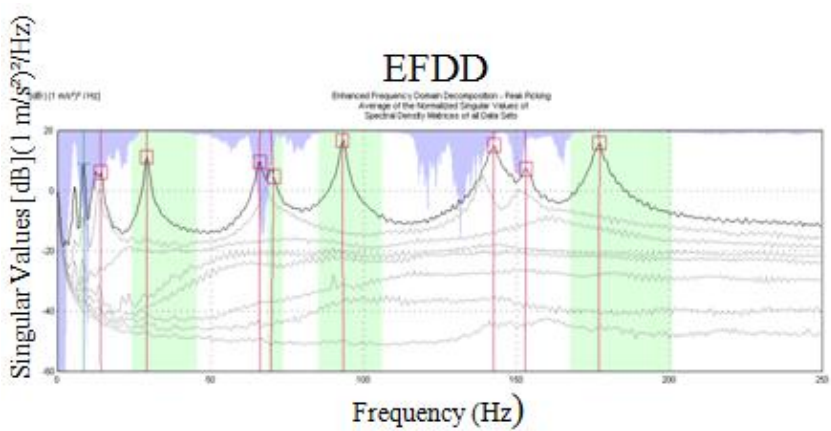

Fig. 4: EFDD Singular Value Decomposition (SVD) Plot

From SVD plot obtained as in Figure 3 and Figure 4, there were multiple modes estimated for frequency range less than $200 \mathrm{~Hz}$ The resonance peaks seem to be well separated. There were 9 peaks representing 9 modes of interest were selected since this study is focused on capturing flexural modes up to 3rd order of bending and torsion for both horizontal (X and $\mathrm{Y}$ ) directions. The frequencies are determined using Single Degree of Freedom (SDOF) in a user-definable frequency band around the peak while mode shapes are determined from singular vector weighted by singular values in the frequency band.

All 14 projection channels were applied for the FDD and EFDD methods. The SVD plots are not significantly affected by the number of projection channels but only its processing time will differ.

However, it is required to compute the SVD plot using all projection channels preliminarily to analyse how many channels could be further remove in SSI techniques in order to obtain better stabilization diagram. It is also best to use all 14 channels for FDD to make sure no important information is lost during signal processing. This is crucial as the FDD method will act as a reference method for the other parametric algorithms.

\subsection{SSI Methods Peak Picking Identification}

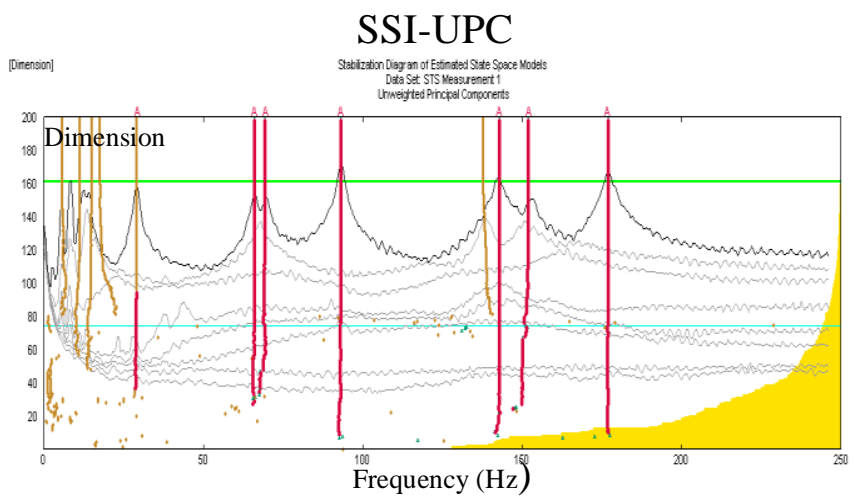

Fig.5: SSI-UPC stabilization diagram.

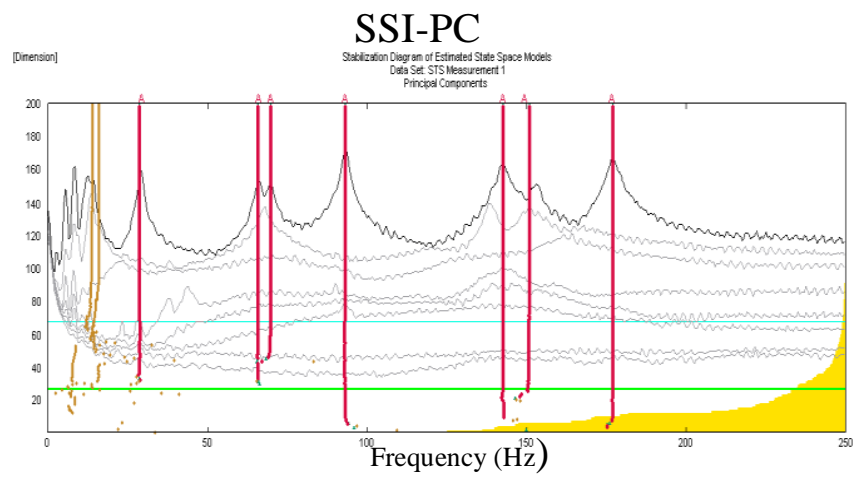

Fig.6: SSI-PC stabilization diagram.

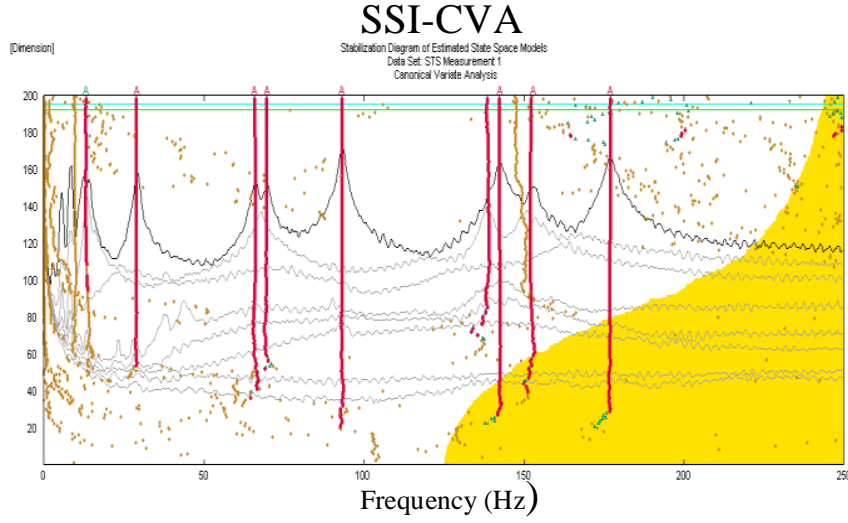

Fig.7: SSI-PC stabilization diagram.

Projection channel applied for the SSI analysis was determined by studying SVD plot where 8 projection channels were sufficient to remove redundant information without introducing local modes. SSI methods in comparison to FDD and EFDD were much more significantly affected from the number of projection channels as it was found that when all channels were used, it produced many noise modes. In the presence of high noisy modes, it becomes difficult for determining modal domain of real physical deformation.

SSI-UPC were able to detect first mode in the lower frequencies as compared to SSI-PC as shown in Figure 5 and Figure 6. However, the mode obtained from SSI-UPC shows first mode as a noise mode. This is reasonably accepted as correlation of the first mode shape from SSI-UPC shows agreement with the mode shape obtained from the FDD method.

SSI-CVA in Figure 7 displayed plenty of scattered noise modes at higher frequency ranges. This can be reduced by using different projection channels but not opted in this study as it might introduce local modes. Setting state space dimension from 0-200 was sufficient to detect stable modes for frequencies less than $200 \mathrm{~Hz}$.

\subsection{Natural Frequencies}

From SVD plots and SSI stability diagrams depicted in Figure 3 to Figure 7, the resulting natural frequencies and visual mode shapes for FDD were presented in Table 1 and the comparison of natural frequencies for both non-parametric method (FDD algorithm) and parametric methods (EFFD and SSI algorithms) is illustrated in Table 2.

The results present in this section indicate that all methods applied in this study have produced the correlated modal data. Good experimental modal data from FDD is useful for further application after it has been compared and validated with other techniques. This comparison is important to ensure that the structure natural frequencies and mode shapes are actually related to the structure physical information and not from other sources.

\subsection{Visual Mode Shapes (FDD)}

Table 1: Structure mode shapes using FDD

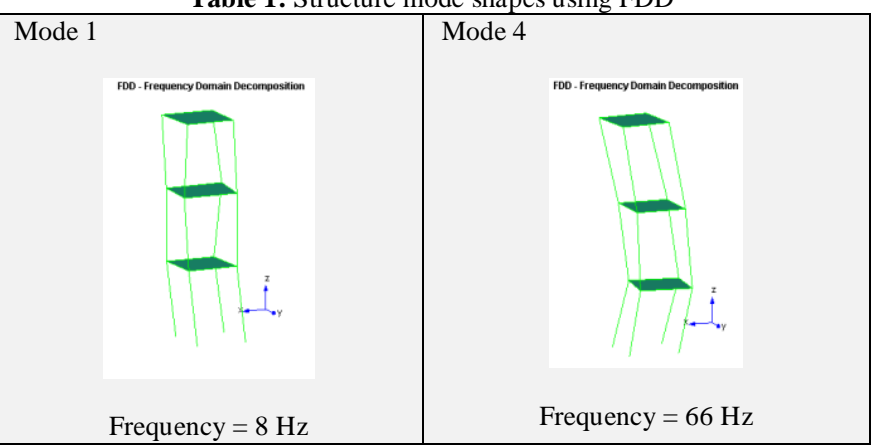




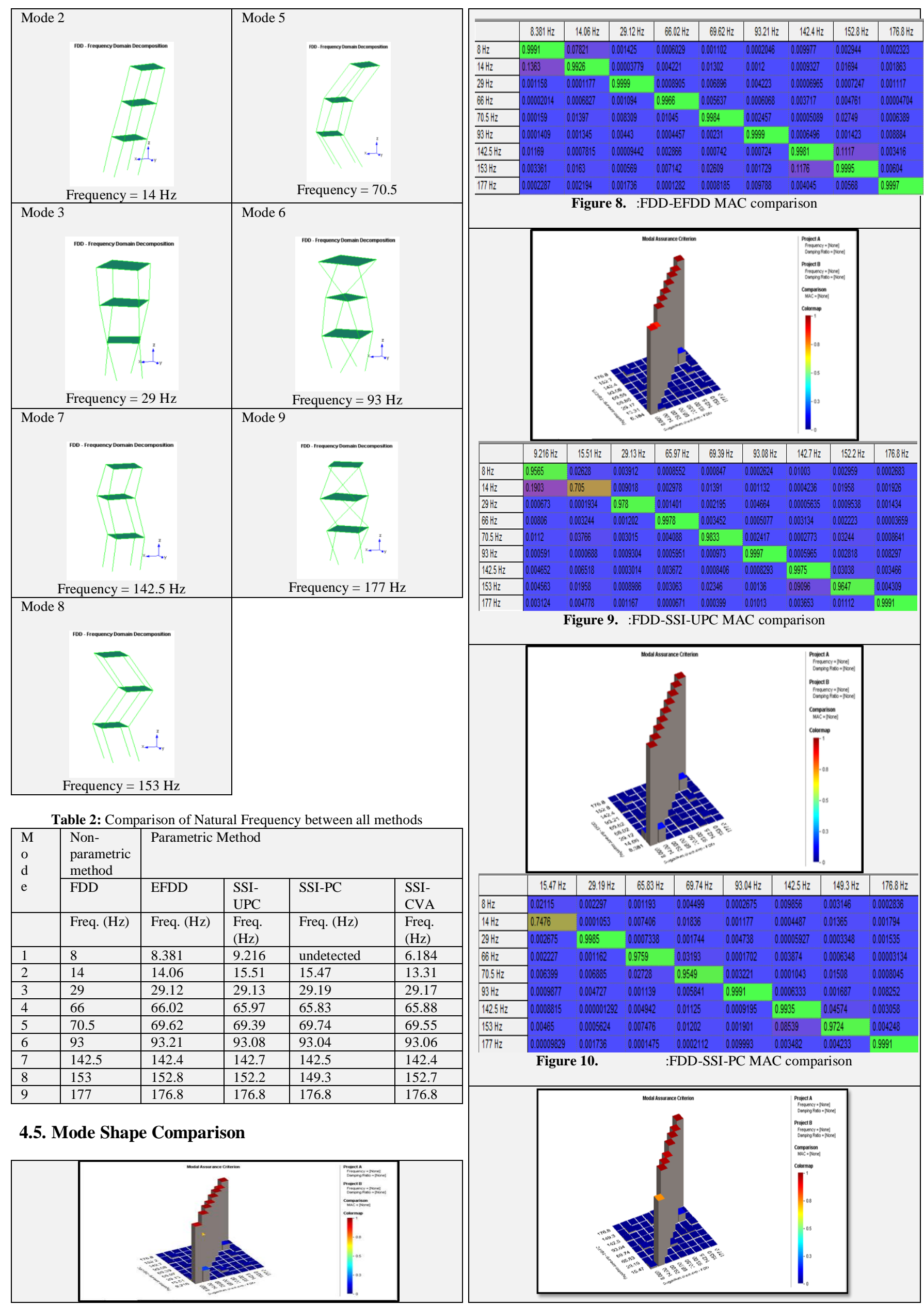




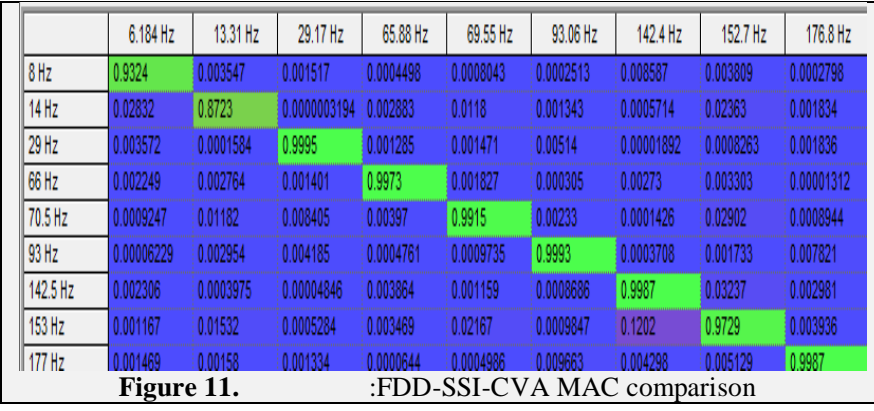

Modal Assurance Criterion (MAC) analysis was used as a statistical indicator for mode validation to indicate the correlation between mode shapes which provides additional confidence factor in the evaluation of a modal vector from different modal parameter estimation algorithms. MAC values plotted in Figure 8 to Figure 11 exhibit good correlation for FDD-EFDD and FDD-SSI across all 9 modes. However, for SSI algorithms, the MAC values for lower modes especially for the second mode was relatively low when compared with FDD. Both UPC and PC methods showed MAC value of around 0.75 . For the SSI CVA, mode 2 exhibited higher MAC value than other SSI algorithms but was still less than the desired range of $0.9-1$ with 0.87 correlation.

\section{Conclusion}

In this paper, Operational Modal Analysis (OMA) was successfully carried out and a 3D Scaled Model of a 3-Storey Aluminium Structure which undergoing random excitation. All the objectives of this study have been achieved as the modal parameters of the structure under the designated boundary condition (clamped at the base) were extracted. Good modal parameters were determined from both parametric and non-parametric methods in OMA techniques. Natural frequencies from FDD, EFDD and SSI-CVA methods were nearly close to the FDD method. Moreover, MAC values showed that mode shapes from EFDD and CVA methods are well correlated with that of FDD method. In general, the results of this study indicate a strong correlation of modal parameters for the 3D Scaled Model of a 3-Storey Aluminium Structure using both parametric and non-parametric methods.

It is recommended that the research be extended to investigate the dynamic characteristics of the same structure using Finite Element Analysis (FEA). Furthermore, finite element updating can also be performed by using the a discrepancy result between FEA and test (OMA) to correct errors in FE models for further structural vibration analysis with much more confidence.

\section{References}

[1] M. M. Derriso, C. D. McCurry, and C. M. Schubert Kabba (2016), Structural Health Monitoring (SHM) in Aerospace Structures., Elsevier,

[2] A. Gómez González and S. D. Fassois (2016), A Supervised Vibration-Based Statistical Methodology for Damage Detection under Varying Environmental Conditions \& its Laboratory Assessment with a Scale Wind Turbine Blade, J. Sound Vib., vol. 366, pp. 484500 ,

[3] M. Almeida, P. Singhal, A. Sequeira, R. Church, and V. Srivastava (2015), An Integrated System For Health Monitoring Of Civil Infrastructures Using A Sensor Network, IEEE Canada International Humanitarian Technology Conference (IHTC2015), pp. 1-4.

[4] D. Liang and S. Yuan (2015), Structural Health Monitoring System Based On Multi-Agent Coordination And Fusion For Large Structure, Adv. Eng. Softw., vol. 86, pp. 1-12,

[5] F. Lorenzoni, F. Casarin, M. Caldon, K. Islami, and C. Modena (2015), Uncertainty Quantification in Structural Health Monitoring: Applications on Cultural Heritage Buildings, Mech. Syst. Signal Process. vol. 66, pp. 268-281.
[6] Z. W. Chen, Q. L. Cai, and J. Li, (2016), Stress Influence Line Identification Of Long Suspension Bridges Installed With Structural Health Monitoring Systems, Int. J. Struct. Stab. Dyn, vol. 16, no. 04, p. 1640023

[7] C. Chisari, C. Bedon, and C. Amadio (2015), Dynamic And Static Identification Of Base-Isolated Bridges Using Genetic Algorithms, Eng. Struct. vol. 102, pp. 80-92.

[8] G. Comanducci, F. Ubertini, and A. L. Materazzi (2015), Structural Health Monitoring Of Suspension Bridges With Features Affected By Changing Wind Speed, J. Wind Eng. Ind. Aerodyn., vol. 141, pp. 12-26.

[9] M. T. Yarnold and F. L. Moon (2015), Temperature-Based Structural Health Monitoring Baseline For Long-Span Bridges, Eng. Struct., vol. 86, pp. 157-167.

[10] H. A. G.F. Sirca Jr. (2012, System Identification in Structural Engineering.

[11] M. Batel, G. S., M. N., and H. Herlufsen (2003), Ambient Response Modal Analysis on A Plate Structure, in Proceedings of 21st International Modal Analysis Conference (IMAC-XXI).

[12] M. Goursat, M. Döhler, L. Mevel, and P. Andersen (2011), Crystal Clear SSI For Operational Modal Analysis of Aerospace Vehicles, in Structural Dynamics, Volume 3 SE - 125, T. Proulx, Ed. Springer New York, pp. 1421-1430.

[13] A. Skafte and R. Brincker (2013), Estimation Of Unmeasured DOF's On A Scaled Model Of A 4-Storey Building, Conference Proceedings of the Society for Experimental Mechanics Series, vol. 6, pp. 347-353.

[14] R. Brincker and C. E. Ventura (2015), Introduction to Operational Modal Analysis. 